\title{
Severe acute kidney injury in critically ill COVID-19 patients
}

\author{
Gaston J. Piñeiro ${ }^{1}(1)$. Alicia Molina-Andújar ${ }^{1}$ - Evelyn Hermida ${ }^{1}$ Miquel Blasco ${ }^{1}$ Luis F. Quintana ${ }^{1}$. \\ Guido Muñoz Rojas ${ }^{2} \cdot$ Jordi Mercadal $^{2} \cdot$ Pedro Castro $^{3} \cdot$ Elena Sandoval $^{4} \cdot$ Rut Andrea $^{5} \cdot$ Javier Fernández $^{6}$. \\ Joan Ramon Badia ${ }^{7}$ Alex Soriano ${ }^{8}$. Esteban Poch ${ }^{1}$ on behalf of the Hospital Clínic Critical Care COVID-19 working \\ group (CCCC)
}

Received: 15 July 2020 / Accepted: 12 November 2020 / Published online: 2 January 2021

(C) Italian Society of Nephrology 2021

\begin{abstract}
Background Acute kidney injury (AKI) is frequent in Coronavirus Infection Disease 2019 (COVID-19) patients. Factors associated with AKI in COVID-19 intensive care unit (ICU) patients and their outcomes have not been previously explored. Methods Prospective observational study of COVID-19 patients admitted to the ICUs of the Hospital Clínic of Barcelona (Spain), from March 25th to April 21st, 2020, who developed AKI stage 2 or higher (AKIN classification). The primary goal was to describe the characteristics of moderate-severe AKI of COVID-19 patients in an ICU context. As a secondary goal, we aimed to find independent predictors of AKI progression, Renal Replacement Therapy (RRT) requirement and mortality among these patients.

Results During the study period, 52 out of 237 ICU patients, developed AKIN stage 2 or higher and were included in the study. A Sequential Organ Failure Assessment (SOFA) score at AKI diagnosis of 8 or higher was associated with RRT, OR 5.2, p 0.032. At the time of AKI diagnosis, patients had a worse liver profile and higher inflammation markers than at admission. Fifty per cent of the patients presented AKI progression from AKIN 2 to 3 and $28.85 \%$ required RRT. The use of corticosteroids in $69.2 \%$ of patients was associated with a reduced requirement of RRT, OR 0.13 (CI 95\% 0.02-0.89), p 0.037 . AKI was associated with high mortality (50\%) and a longer hospital stay, median 35 vs 18 days (p 0.024).

Conclusions The prevalence of moderate/severe AKI in COVID-19 patients admitted to the ICU is high and has a strong correlation with mortality and length of hospital stay.
\end{abstract}

Keywords COVID-19 $\cdot$ Acute kidney injury $\cdot$ Intensive care $\cdot$ Renal replacement therapy

\section{Introduction}

The outbreak of the new Coronavirus Infection Disease 2019 (COVID-19) has rapidly evolved into a global pandemic with devastating consequences $[1,2]$. On May 20th,

Gaston J. Piñeiro and Alicia Molina-Andújar equally contributed.

The Collaborator names of the Hospital Clínic Critical Care COVID-19 working group (CCCC) is given in the Acknowledgements section.

Supplementary Information The online version contains supplementary material available at https://doi.org/10.1007/s4062 0-020-00918-7.

Gaston J. Piñeiro

gjpineir@clinic.cat

Extended author information available on the last page of the article
2020, Spain had 232,555 patients affected by COVID-19 and ranked 5th in the world for most positive cases [3]. At the Hospital Clínic of Barcelona, the first case was detected on February 25th, and from then until May 21st, 2,418 patients were admitted to the institution, of whom, 444 (18.36\%) required intensive care unit (ICU) admission.

COVID-19 patients predominantly develop respiratory symptoms, and in some patients, it evolves to acute respiratory distress syndrome (ARDS) or multi-organ failure, including kidney injury [3]. Severe Acute Respiratory Syndrome Coronavirus 2 (SARS-CoV-2) infects the host using the angiotensin-converting enzyme 2 receptor (ACE2) that is expressed in several organs, including the kidney [4-7]. Acute kidney injury (AKI) is frequent in COVID-19; reports from China, Italy, and the USA found a rate of AKI that ranges widely from 0.5 to $36.6 \%$ in hospitalized patients, 
and it portends poor prognosis [8-16]. As for critically ill patients, the described incidence was 19-23\% [17, 18].

Several contributors have been proposed in the pathogenesis of AKI in patients with COVID-19: immunologic injury associated with inflammatory response, direct cytotoxicity over endothelial and tubuloepithelial cells and podocytes, microthrombi and thrombotic microangiopathy, or cardiorenal syndrome due to right ventricular failure may be present $[19,20]$.

Factors associated with AKI in COVID-19 ICU patients and their outcomes have not been previously explored. The objective of our study is to describe the characteristics of moderate-severe AKI in the ICU context. As a secondary objective, we attempted to find independent predictors of AKI progression to renal replacement therapy (RRT) requirement and mortality.

\section{Methods}

This is a prospective observational study. SARS-CoV-2 infection was diagnosed by reverse transcription-quantitative PCR (RT-PCR) assay of nasopharyngeal swab specimens. We included all the patients with COVID-19 who developed AKI $\geq 2$ according to AKIN classification [21], and who were admitted to the ICUs of the Hospital Clínic of Barcelona (Spain) from March 25th to April 21st, 2020. The exclusion criteria were: chronic dialysis treatment, kidney transplant, or chronic kidney disease (CKD) KDIGO stage 4 (estimated glomerular filtrate rate (eGFR) less than $30 \mathrm{ml} /$ min) [22]. The patients were followed-up until discharge from hospital, death, or the last follow-up day (July 15th, 2020).

Demographic variables, including Body Mass Index (BMI), date of illness onset and symptoms, vital signs, blood test studies and Sequential Organ Failure Assessment (SOFA) score (at hospital admission, at AKI stage 2 diagnosis, and at RRT start), use of vasoactive drugs, pharmacological treatments received, use of mechanical ventilation, RRT characteristics, urinary output at AKI stage 2, date of RRT start, date of ICU/hospital discharge and mortality were recorded. Comorbidities, which included cardiovascular disease, current or previous history of smoking, hypertension, diabetes, chronic obstructive pulmonary disease (COPD), human immunodeficiency virus (HIV) infection, and presence of active neoplasm, were also assessed, as were angiotensin-converting enzyme inhibitor (ACEI) and angiotensin receptor blocker (ARB) treatment at baseline. The suspected cause of AKI was evaluated; COVID-19-related AKI was considered if no other explanation was evident or suspected after diagnostic workup. RRT indication, modality and dose were based on clinical judgment.
In general, patients with hemodynamic stability and use of vasopressors at a dose lower than $0.1 \mathrm{mcg} / \mathrm{kg} / \mathrm{min}$ received intermittent hemodialysis, according to our protocol. The choice between continuous hemodialysis with medium cutoff or continuous hemodiafiltration was based on logistic criteria and the experience of each intensive care unit within the hospital.

AKI recovery was defined if last creatinine level was less than twice that at baseline.

Overall mortality and length of stay (LOS) among patients with COVID-19 diagnosis admitted to the ICU during the study period, and without exclusion criteria, were collected and compared with the cohort of patients with AKI.

\section{Statistical analysis}

Data were described as mean \pm standard deviation (SD) if normally distributed, or median and interquartile range otherwise. Categorical variables were expressed as absolute and relative (\%) frequencies. Group comparisons were made by Student's $t$ test, Logistic regression, Fisher's exact $t$ test or Mann-Whitney $U$ test for independent groups when appropriate. Statistical associations were explored univariately, and all $\mathrm{p}$ values $<0.05$ were included in the multivariate analysis. Statistical analyses were performed using IBM SPSS statistics V25.0 software (IBM Corp, Armonk, NY, USA). A two-sided $p$ value $\leq 0.05$ was used to indicate statistical significance.

\section{Results}

\section{General characteristics}

Two-hundred and thirty-seven patients with COVID-19 and without exclusion criteria required ICU admission. Fifty-two (21.4\%) patients presented AKI $\geq 2$ of the AKIN classification and were included in the study. The median follow-up was 35.5 (IQR 36.75) days. Table 1 summarizes the demographic and clinical characteristics of the patients at hospital admission.

Patients were mostly male (76.9\%), with a median age of 71.5 (61-74.75) years. Regarding comorbidities, $73.08 \%$ of the patients presented at least one, with hypertension, diabetes and COPD being the most frequent $(59.6 \%, 21.2 \%$ and $21.2 \%$, respectively). Overweight was present in $17 \%$ of the patients, and $13.5 \%$ presented CKD stage 3 . Twenty-one per cent of the patients $(n=11)$ were on treatment with ACEI, and $15.4 \%$ with $\mathrm{ARBs}$ at admission. Both antihypertensive drugs were discontinued in all patients at admission.

The median time from symptom onset to hospital and ICU admission were 7 (IQR 5) and 10 (IQR 4) days, 
Table 1 Baseline characteristics of patients at hospital admission

\begin{tabular}{|c|c|}
\hline & All patients $(n=52)$ \\
\hline Age (years), median (IQR) & $71.5(13.75)$ \\
\hline Sex (male) & $40(76.9 \%)$ \\
\hline \multicolumn{2}{|l|}{ Comorbidities } \\
\hline None & $14(26.9 \%)$ \\
\hline Hypertension & $31(59.6 \%)$ \\
\hline Diabetes & $11(21.2 \%)$ \\
\hline Cardiac failure & $2(3.8 \%)$ \\
\hline Ischemic heart disease & $3(5.8 \%)$ \\
\hline $\mathrm{BMI} \geq 30 \mathrm{~kg} / \mathrm{m}^{2}$ & $9(17.3 \%)$ \\
\hline COPD & $11(21.2 \%)$ \\
\hline Active smoker & $3(5.8 \%)$ \\
\hline Active cancer & $3(5.8 \%)$ \\
\hline HIV infection & $1(1.9 \%)$ \\
\hline CKD III & $7(13.5 \%)$ \\
\hline \multicolumn{2}{|l|}{ Clinical characteristics at admission } \\
\hline Fever & $44(84.6 \%)$ \\
\hline Pneumonia & $49(94.25 \%)$ \\
\hline Dyspnea & $39(75.5 \%)$ \\
\hline $\mathrm{FiO}_{2}(\%)$, median $(\mathrm{IQR})$ & $35(39)$ \\
\hline $\mathrm{SatO}_{2}(\%)$, median (IQR) & $95(6)$ \\
\hline $\mathrm{PaO}_{2} / \mathrm{FIO}_{2}$, mean $\pm \mathrm{SD}$ & $243 \pm 155$ \\
\hline SOFA, mean \pm SD & $5.78 \pm 3.26$ \\
\hline Days from onset to hospital admission, median (IQR) & $7(5)$ \\
\hline Days from onset to ICU, median (IQR) & $10(4)$ \\
\hline ACEIs & $11(21.2 \%)$ \\
\hline $\mathrm{ARBs}$ & $8(15.4 \%)$ \\
\hline Mean baseline creatinine (mg/dL), median (IQR) & $0.96(0.43)$ \\
\hline Median baseline eGFR CKD-EPI ( $\mathrm{ml} / \mathrm{min})$, mean $\pm \mathrm{SD}$ & $76.52 \pm 20$ \\
\hline
\end{tabular}

Median $(\mathrm{IQR}) / \mathrm{n}(\%) /$ mean $\pm \mathrm{SD}$

$B M I$ Body Mass Index, COPD chronic obstructive pulmonary disease, HIV human immunodeficiency virus, $C K D$ chronic kidney disease, ACEIs angiotensin converting enzyme inhibitors, ARBs angiotensin II receptor blockers, $\mathrm{PaFiO}_{2}$ arterial blood oxygen pressure, $\mathrm{FiO}_{2}$ fraction of inspired oxygen, $\mathrm{MV}$ mechanical ventilation, SOFA Sequential Organ Failure Assessment score, $e$ GFR Estimated Glomerular Filtration Rate respectively. Pneumonia was present in $94.2 \%$ of cases, with a mean $\mathrm{PaO}_{2} / \mathrm{FiO}_{2}$ of $243 \pm 155 \mathrm{mmHg}$, and a mean SOFA at admission of $5.78 \pm 3.26$.

Table 2 summarizes the laboratory findings. At the time of AKI diagnosis, patients had a worse liver profile, higher levels of D-dimer, procalcitonin, leukocytes, and platelets than at admission. C-Reactive Protein (CRP) levels were lower at AKI diagnosis compared to admission values. SOFA scores also showed a significant increase between admission and AKI diagnosis (5.79 \pm 3.26 and 8.38 \pm 2.43 , respectively, $\mathrm{p}<0.01)$.

Thrombotic events occurred in ten patients (19.23\%): eight pulmonary thromboembolisms and two peripheral arterial occlusions. On the other hand, hemorrhagic complications occurred in 11 patients $(21.15 \%)$ : four gastrointestinal bleeds, three retroperitoneal bleeds, three muscle and one central nervous system bleeds.

\section{Treatment}

Most of the patients received therapy with the combination of hydroxychloroquine, azithromycin (96.2\%), and lopinavir/ritonavir (94.2\%). Steroids were administered to $69.2 \%$ of the patients with a mean cumulative dose of $544 \pm 496.38 \mathrm{mg}$ of prednisone. Tocilizumab was used in $48.1 \%$ of cases; the median time between admission and tocilizumab administration was two days (1-4), mean dose $561 \pm 276.24$. Five $(9.6 \%)$ patients with refractory inflammatory response were treated with Anakinra.

Regarding antithrombotic treatment, $5(9.6 \%)$ patients were anticoagulated, $38(73 \%)$ received antithrombotic prophylaxis, and $9(17.3 \%)$ did not receive antithrombotic therapy at hospital admission. At diagnosis of AKI, 10 $(19.2 \%)$ patients were on anticoagulant treatment, $38(73 \%)$ with prophylaxis and $4(7.7 \%)$ without antithrombotic 
Table 2 Laboratory findings

\begin{tabular}{llcl}
\hline & At admission & At AKI & $\mathrm{p}$ \\
\hline Creatinine (mg/dL) [0.3-1.3] & $1.11(1.12)$ & $2.23(1)$ & 0.001 \\
Hemoglobin $(\mathrm{g} / \mathrm{dL})[12-15]$ & $13.8(32.5)$ & $11.9(3.9)$ & 0.001 \\
WC count $\left(\times 10^{6} / \mathrm{L}\right)[1-4,4-11]$ & $7255(8687)$ & $12,500(11,212.5)$ & 0.001 \\
Lymphocytes $\left(\times 10^{6} / \mathrm{L}\right)[100-1000]$ & $700(400)$ & $700(700)$ & 0.56 \\
Platelets $\left(\times 10^{9} / \mathrm{L}\right)[130-400]$ & $184.5(119)$ & $226.5(174.25)$ & 0.002 \\
Prothrombin time (\%) [80-100] & $68.45(119)$ & $73.1(20.52)$ & 0.43 \\
Sodium $(\mathrm{mmol} / \mathrm{L})[135-145]$ & $138(5)$ & $138.5(6)$ & 0.016 \\
Potassium $(\mathrm{mmol} / \mathrm{L})[3.5-5.5]$ & $3.8(0.8)$ & $4.1(1.07)$ & 0.021 \\
D-Dimer $(\mathrm{ng} / \mathrm{ml})[<500]$ & $1100(4450)$ & $4600(5750)$ & 0.026 \\
Lactate dehydrogenase $(\mathrm{U} / \mathrm{L})[<234]$ & $429(235)$ & $8(18.18)$ & 0.63 \\
C-reactive protein $(\mathrm{mg} / \mathrm{L})[<1]$ & $13.58(42.25)$ & $0.92(3.13)$ & 0.02 \\
Procalcitonin $(\mathrm{ng} / \mathrm{mL})[<0.5]$ & $0.23(1.56)$ & $1(1.4)$ & 0.005 \\
Bilirubin $(\mathrm{mg} / \mathrm{dL})[0.2-1.2]$ & $0.5(0.5)$ & $51.5(57.75)$ & 0.001 \\
AST $(\mathrm{U} / \mathrm{L})[5-40]$ & $56.5(42.25)$ & $53.5(61.75)$ & 0.99 \\
ALT $(\mathrm{U} / \mathrm{L})[5-40]$ & $43(38)$ & $1215(1817)$ & 0.027 \\
Ferritin $(\mu \mathrm{g} / \mathrm{L})[15-200]$ & $\mathrm{N} / \mathrm{D}$ & & \\
\hline
\end{tabular}

Median (IQR)/n (\%)/mean \pm SD

[] Normal values, $W C$ white cells, $A L T$ alanine aminotransferase, $A S T$ aspartate aminotransferase, $N / D$ no data

therapy. Low molecular weight heparin was used in 40 $(76.9 \%)$ and $43(82.69 \%)$ patients at admission and at AKI diagnosis, respectively. Moreover, three and five patients received unfractionated heparin at hospital admission and AKI diagnosis, respectively. One patient received fondaparinux at AKI time because of heparin-induced thrombocytopenia.

\section{AKI characteristics}

AKI characteristics and evolution are described in Table 3. Most of the patients (84.6\%) developed AKI during the ICU stay, within a median of 5 and 12 days from admission and the onset of symptoms, respectively. Twenty-six (50\%) of the cases progressed to more severe AKI.

Patients were mostly non-oliguric at AKI diagnosis, but those who started RRT developed it more frequently (in the $24 \mathrm{~h}$ before RRT onset). Hyperkalemia was not a frequent finding at the time of AKI diagnosis.

At AKI diagnosis, $73 \%$ of the patients were on treatment with vasoactive drugs, with a median noradrenaline dose of $0.19 \mathrm{mcg} / \mathrm{kg} / \mathrm{min}$ (IQR 0.46).

Suspected intrinsic AKI related to severe COVID-19, which accounted for $71.15 \%$ (n 37) of cases, was the most common form of kidney dysfunction. The other probable causes of AKI that were identified included: hemorrhagic shock related to anticoagulation treatment (4); contrast nephropathy (3); drug nephrotoxicity (2); cardiac arrest (2); liver failure (2); heart failure (1) and pancreatitis (1).
Fifteen patients (28.85\%) required RRT, including 13 with continuous renal replacement therapy (CRRT). Median time from admission to RRT was 12 (IQR 10) days. RRT mean time was $10.87 \pm 9.28$ days. CRRT was the only modality in eight patients, while five patients received CRRT followed by intermittent modality, and two patients were treated with intermittent modalities alone. When CRRT was used, the modalities included continuous hemodialysis with medium cut-off dialyzers $(\mathrm{MCO})(\mathrm{n}=5)$ and continuous hemodiafiltration with high-flux hemofilter $(n=8)$. Anticoagulation consisted of citrate in six patients and heparin in six patients.

At the end of follow-up, $31(59.6 \%)$ patients recovered from the AKI episode.

\section{Outcomes}

Variables associated with RRT or mortality are described in Tables 4 and 5 and 6 (Supplementary material).

Baseline and admission creatinine was higher in patients who died, p 0.011 and 0.02, respectively (Table 6 supplementary materials). In univariate analysis, the variables associated with higher mortality in AKI patients were age $>60$ years ( $p$ 0.017), and presence of one or more comorbidities ( $p$ 0.03). On the contrary, recovery from AKI was associated with less probability of death, $p<0.001$. In the multivariate analysis, only AKI recovery retains significance (p 0.005).

Overall mortality among patients with COVID-19 diagnosis admitted to the ICUs during the study period, and 
Table 3 AKI characteristics and evolution of the patients

\begin{tabular}{|c|c|}
\hline & All patients $(n=52)$ \\
\hline AKI present at hospital admission & $8(15.4 \%)$ \\
\hline AKI occurrence during hospital stay & $44(84.6 \%)$ \\
\hline Days from hospital admission to AKI, median (IQR) & $5(9.25)$ \\
\hline Days from symptom onset to AKI, median (IQR) & $12(8-18)$ \\
\hline $\mathrm{Pa} / \mathrm{FIO}_{2}$ at $\mathrm{AKI}$, mean $\pm \mathrm{SD}$ & $209.51 \pm 94.71$ \\
\hline Mechanical ventilation (MV) & $44(84.6 \%)$ \\
\hline Days hospital admission MV, median (IQR) & $1(3)$ \\
\hline $\mathrm{SOFA}$ at $\mathrm{AKI}$, mean $\pm \mathrm{SD}$ & $8.38 \pm 2.43$ \\
\hline Vasoactive drugs at AKI & $38(73.1 \%)$ \\
\hline Noradrenaline dose $\mathrm{mcg} / \mathrm{kg} / \mathrm{min}$, median (IQR) & $0.19(0.459)$ \\
\hline Diuresis $24 \mathrm{~h}$ prior to AKI diagnosis (mL), median (IQR) & $1500(1352)$ \\
\hline Diuresis $24 \mathrm{~h}$ before first RRT diagnosis (mL), median (IQR) & $330(665)$ \\
\hline \multicolumn{2}{|l|}{ AKI stage at diagnosis } \\
\hline Stage 2 & $43(82.7 \%)$ \\
\hline Stage 3 & $9(17.3 \%)$ \\
\hline Renal replacement therapy (RRT) & $15(28.85 \%)$ \\
\hline Days from hospital admission to first RRT, median (IQR) & $12(11)$ \\
\hline RRT days, median (IQR) & $7(17)$ \\
\hline RRT at death & $5(9.6 \%)$ \\
\hline Continuous renal replacement therapy (CRRT) & $13(25 \%)$ \\
\hline CRRT days, mean $\pm \mathrm{SD}$ & $6.77 \pm 3.56$ \\
\hline Intermittent RRT sessions, median (IQR) & $6(3-9)$ \\
\hline AKI progression from AKIN2 to AKIN3/RRT & $26(60.46 \%)$ \\
\hline AKI recovery & $31(59.6 \%)$ \\
\hline Total death & $27(51.92 \%)$ \\
\hline Death with AKI stage 2 & $1(1.92 \%)$ \\
\hline Death with AKI stage 3 & $20(38.5 \%)$ \\
\hline Discharged & $25(48.08 \%)$ \\
\hline
\end{tabular}

Median (IQR)/n (\%)/mean \pm SD

$A K I$ acute kidney injury, $R R T$ renal replacement therapy, $C R R T$ continuous renal replacement therapy, IQR interquartile range, $S D$ standard deviation, GFR glomerular filtration rate, SOFA Sequential Organ Failure Assessment score, $\mathrm{PaFiO}_{2}$ arterial blood oxygen pressure, $\mathrm{MV}$ mechanical ventilation without exclusion criteria, $n=237$, was $16.87 \%(n=40)$. In the non-AKI patients, mortality was $7.3 \%(n=13)$, while in AKI patients it was $51.92 \%(\mathrm{n}=27), \mathrm{p}<0.001$, OR 10.67 (95\% CI 5.1-22.33). The median time to death after AKI diagnosis was eight days (IQR 13). AKI was also associated with longer hospital LOS, 35 (IQR 36.75) vs 18 (IQR 20) days in AKI vs non-AKI patients, respectively (p 0.018)).

Regarding RRT, in the univariate analysis, a SOFA score of 8 or more at AKI diagnosis was associated with the need for RRT, p 0.032, OR 4.52, with sensitivity and specificity of $73 \%$ and $62 \%$, respectively. However, the non-renal SOFA score at AKI was not associated with RRT. Also the use of vasoactive drugs at AKI diagnosis was not associated with RRT requirement, $\mathrm{p}$ 0.06. On the contrary, the use of corticosteroids was associated with less requirement of RRT, p 0.03 , OR 0.24.
In the multivariate analysis, a SOFA score of 8 or more and corticosteroid use at AKI diagnosis were associated with RRT, OR 6.15, p 0.015, and OR 0.16, p =0.017 respectively.

\section{Discussion}

Kidney involvement is frequent in COVID-19. Cheng et al. described kidney abnormalities in more than $40 \%$ of COVID-19 patients in Wuhan (China), including proteinuria, hematuria and elevated serum creatinine [14]. Also, any degree of AKI has been associated with mortality in COVID-19 hospitalized patients [9-11, 13-15, 17, 23]. In this prospective observational single-center study, moderatesevere AKI was associated with high mortality (50 vs $7.3 \%$ ) and hospital length of stay (35 vs 18 days). 
Table 4 Univariate and multivariate analysis of clinical characteristics and treatment related to RRT

\begin{tabular}{|c|c|c|c|c|}
\hline & \multicolumn{2}{|l|}{ Univariate } & \multicolumn{2}{|l|}{ Multivariate } \\
\hline & OR (CI 95\%) & $\mathrm{p}$ & OR (CI 95\%) & $\mathrm{p}$ \\
\hline Male (yes) & $0.76(0.19-3.03)$ & 0.7 & - & - \\
\hline Age $>60$ years & $5.18(0.6-44.72)$ & 0.13 & - & - \\
\hline Hypertension history & $0.7(0.21-2.34)$ & 0.56 & - & - \\
\hline Diabetes & $0.91(0.2-4.01)$ & 0.89 & - & - \\
\hline COPD & $0.19(0.02-1.66)$ & 0.13 & - & - \\
\hline CKD III & $0.98(0.17-5.73)$ & 0.99 & - & - \\
\hline $\mathrm{BMI}>30$ & $0.6(0.1-3.51)$ & 0.57 & - & - \\
\hline Presence of one or more comorbidities & $1.56(042-5.77)$ & 0.51 & - & - \\
\hline ARB history & $0.31(0.03-2.73)$ & 0.29 & - & - \\
\hline ACEI history & $0.48(0.09-2.54)$ & 0.39 & - & - \\
\hline $\mathrm{SOFA} \geq 8$ at $\mathrm{AKI}$ & $4.52(1.2-16.97)$ & 0.03 & $6.5(1.43-29.82)$ & 0.015 \\
\hline Non-renal $\mathrm{SOFA} \geq 7$ at $\mathrm{AKI}$ & $2.07(0.58-7.44)$ & 0.26 & - & - \\
\hline Mechanical ventilation & $3.27(0.37-29.16)$ & 0.29 & - & - \\
\hline PA/FIO2 admission $<200 \mathrm{mmHg}$ & $0.58(0.16-2.13)$ & 0.41 & - & - \\
\hline PA/FIO2 at AKI < $200 \mathrm{mmHg}$ & $2.04(0.59-7.05)$ & 0.26 & - & - \\
\hline AKIN 3 at diagnosis time & $4.12(0.93-18.36)$ & 0.05 & - & - \\
\hline Vasoactive drugs at AKIN & $7.58(0.89-64.33)$ & 0.06 & - & - \\
\hline Corticosteroids & $0.24(0.07-0.87)$ & 0.03 & $0.16(0.04-0.73)$ & 0.017 \\
\hline Tocilizumab & $1.21(0.36-4)$ & 0.76 & - & - \\
\hline Lopinavir/Ritonavir & $0.91(0.83-0.35)$ & 0.26 & - & - \\
\hline Thrombotic event & $1.07(0.24-4.85)$ & 0.93 & & \\
\hline
\end{tabular}

$A K I$ acute kidney injury, $R R T$ renal replacement therapy, $C R R T$ continuous renal replacement therapy, $I Q R$ interquartile range, $S D$ standard deviation, GFR glomerular filtration rate, SOFA Sequential Organ Failure Assessment score, $\mathrm{PaFiO}_{2}$ arterial blood oxygen pressure, $\mathrm{MV}$ mechanical ventilation, $\mathrm{BMI}$ Body Mass Index, $C O P D$ chronic obstructive pulmonary disease, $H I V$ human immunodeficiency virus, $C K D$ chronic kidney disease, ACEIs angiotensin converting enzyme inhibitors, ARBs angiotensin II receptor blockers, $\mathrm{PaFiO}_{2}$ arterial blood oxygen pressure, $\mathrm{FiO}_{2}$ fraction of inspired oxygen
Several mechanisms of kidney damage have been described, but many facts point to direct damage. Low counts of SARS-CoV-2 virus have been detected in kidney cells [20]; furthermore, SARS-CoV-2 infects the host using the ACE2 receptor, transmembrane serine protease 2, and cathepsin L, all are enriched in kidney tissue [24]. Even more endothelial cells express ACE2 receptors, and direct infection of SARS-CoV-2 on engineered human blood vessel organoids has been described [25]. Kidney histology in patients with COVID-19 has shown the presence of acute tubular necrosis, and moderate-to-severe lymphocytic infiltration and collapsing glomerulopathy $[12,20,26]$. The virus could also initiate CD68 + macrophage infiltration together with complement $\mathrm{C} 5 \mathrm{~b}-9$ deposition to mediate tubular damage [12].

In our cohort, AKI episodes occurred together with worsening respiratory symptoms (median time from admission to AKI of 5 days), which supports either a direct mechanism of damage to the kidney or an immune-mediated one. In this sense, patients had higher bilirubin, D-dimer, and platelets at AKI diagnosis compared to the admission levels, supporting the hypothesis that severe AKI is part of multiorgan failure that occurs in the context of the cytokine storm that has been described in these patients. In accordance with this, a SOFA score of 8 or more at the time of AKI diagnosis was associated with higher RRT requirement. Also, procalcitonin was higher at the time of AKI diagnosis, which could be related to an inadvertent bacterial co-infection. Bacterial over infection cannot be ruled out as a worsening factor in AKI patients. However, it was not possible to confirm this overlap beyond a suspected diagnosis in most patients.

The treatment protocol for COVID-19 in our center included antiviral therapy with lopinavir/ritonavir, hydroxychloroquine and azithromycin. If patients showed clinical deterioration then biological therapy, which in the majority of cases included an anti-IL-6 (tocilizumab), an anti-IL-1 (anakinra) and/or corticosteroids, was recommended. In our cohort, the inflammatory response was treated with corticosteroids and tocilizumab in $69.2 \%$ and $48.1 \%$ of cases, respectively, at a median time of two days after admission. The use of corticoids was associated with a $13 \%$ reduction in RRT requirement.

In our cohort, $19.23 \%$ of patients presented a thrombotic event, confirming the high incidence reported in the 
Table 5 Univariate and multivariate analysis of clinical characteristics and treatment related to in-hospital mortality

\begin{tabular}{|c|c|c|c|c|}
\hline & \multicolumn{2}{|l|}{ Univariate } & \multicolumn{2}{|l|}{ Multivariate } \\
\hline & OR (CI 95\%) & $\mathrm{p}$ & OR (CI 95\%) & $\mathrm{p}$ \\
\hline Male (yes) & $1.1(0.3-4.02)$ & 0.88 & - & - \\
\hline Age $>60$ years & $7.03(1.34-36.82)$ & 0.017 & $4.16(0.65-26.6)$ & 0.13 \\
\hline Hypertension history & $1.85(0.6-5.66)$ & 0.28 & - & - \\
\hline Diabetes & $3.09(0.72-13.32)$ & 0.17 & - & - \\
\hline COPD & $3.08(0.72-13.3)$ & 0.182 & - & - \\
\hline CKD III & $6.86(0.76-61.67)$ & 0.1 & - & - \\
\hline $\mathrm{BMI}>30$ & $0.5(0.1-2.41)$ & 0.46 & - & - \\
\hline Presence of one or more comorbidities & $4.52(1.2-16.97)$ & 0.03 & $3.29(0.69-15.46)$ & 0.13 \\
\hline ARB history & $1.67(0.35-7.84)$ & 1 & - & - \\
\hline ACEI history & $1.84(0.47-7.25)$ & 0.38 & - & - \\
\hline $\mathrm{SOFA} \geq 8$ at $\mathrm{AKI}$ & $2.58(0.8435-7.93)$ & 0.09 & - & - \\
\hline Non-renal SOFA $\geq 7$ at AKI & $1.1(0.3-4.02)$ & 0.88 & - & - \\
\hline Mechanical ventilation & $0.6(0.13-2.82)$ & 0.7 & - & - \\
\hline PA/FIO2 admission $<200 \mathrm{mmHg}$ & $0.42(0.12-1.47)$ & 0.17 & - & - \\
\hline $\mathrm{PA} / \mathrm{FIO} 2$ at $\mathrm{AKI}<200 \mathrm{mmHg}$ & $0.85(0.27-2.63)$ & 0.77 & - & - \\
\hline AKIN 3 at diagnosis time & $0.4(0.09-1.79)$ & 0.28 & - & - \\
\hline RRT (yes) & $1.08(0.32-3.6)$ & 0.89 & - & - \\
\hline RRT more than 7 days & $1.25(0.16-9.92)$ & 1 & - & - \\
\hline AKI recovery & $0.11(0.03-0.42)$ & 0.001 & $0.13(0.03-0.54)$ & 0.005 \\
\hline Vasoactive drugs at AKIN & $0.75(0.22-2.58)$ & 065 & - & - \\
\hline Corticosteroids & $1.12(0.34-3.63)$ & 0.85 & - & - \\
\hline Tocilizumab & $0.63(0.21-1.88)$ & 0.4 & - & - \\
\hline Lopinavir/Ritonavir & $0.52(0.04-6.13)$ & 1 & - & - \\
\hline Thrombotic event & $0.55(0.13-2.24)$ & 0.49 & - & - \\
\hline
\end{tabular}

$A K I$ acute kidney injury, $R R T$ renal replacement therapy, $C R R T$ continuous renal replacement therapy, $I Q R$ interquartile range, $S D$ standard deviation, GFR glomerular filtration rate, SOFA Sequential Organ Failure Assessment score, $\mathrm{PaFiO}_{2}$ arterial blood oxygen pressure, $\mathrm{MV}$ mechanical ventilation, $B M I$ Body Mass Index, $C O P D$ chronic obstructive pulmonary disease, $H I V$ human immunodeficiency virus, $C K D$ chronic kidney disease, ACEIs angiotensin converting enzyme inhibitors, ARBs angiotensin II receptor blockers, $\mathrm{PaFiO}_{2}$ arterial blood oxygen pressure, $\mathrm{FiO}_{2}$ fraction of inspired oxygen literature $[15,27,28]$. Antithrombotic prophylaxis or anticoagulation was initiated in most of the patients. It is possible that microthrombi and thrombotic microangiopathy can contribute to the pathogenesis of AKI in patients with COVID-19 [15], but given the low number of cases that did not receive these treatments in our study, associations with AKI outcome could not be established. Nevertheless, we want to emphasize the relatively high incidence of major bleeding complications (21.15\%). Patients with impaired kidney function have an increased risk of bleeding, and therefore, the risk/benefit of thromboprophylaxis must be balanced.

The treatments used in our cohort may have altered the course of AKI as the number of patients who required RRT in the present study is relatively low $(15 / 237,6 \%)$ compared to other recent cohorts with an incidence of RRT in ICU patients between 17 and 51\% [12, 13, 16, 17, 23, 29, 30].

The present study has some limitations. First, given the observational nature of the study, bias cannot be ruled out in the beneficial effect of the corticosteroids. Second, the limited number of patients with RRT makes it challenging to come to conclusions about the best strategy for dialyzing patients. Also, the usefulness of MCO in the COVID19 cytokine release syndrome needs further investigation in randomized studies [12].

An important point to consider is that all the patients with CKD KDIGO stage 4 were excluded from the present study to more clearly demonstrate the impact and evolution of AKI in the context of severe COVID-19. Therefore, these results cannot be extrapolated for patients with severe chronic kidney disease.

Given the observational nature of the study, there are no details on proteinuria and hematuria since they were not generally evaluated in our center during the pandemic. There is also no histological material, which would be very useful to describe the type of injury in AKI associated with COVID-19. 
In conclusion, in our study the prevalence of moderate/ severe AKI in COVID-19 in the ICUs is associated with high mortality and hospital length of stay. Present data suggest that corticosteroid treatment may be related to less RRT, but further research is needed to confirm these data.

Acknowledgements The Collaborator names on behalf of the Hospital Clínic Critical Care COVID-19 working group (CCCC) are A. Almuedo, JR Alonso, R. Andrea, F. Aziz, JR. Badia, E. Barbeta, X. Borrat, E. Bragulat, I. Carmona, M. Castellà, P. Castro, O. De Diego, M. Farrero, J. Fernández, S. Fernández, C. Ferrando, M. Ferrer, M. Forga, E. Guasch, $\mathrm{M}^{\mathrm{a}}$ Hernández-Tejero, A. Jacas, P. Leyes, T. López, JA. Martínez, G. Martínez-Palli, R. Mellado, J. Mercadal, G. Muñoz, J. Muñoz, R. Navarro, JM Nicolás, J. Ortiz, E. Poch, M. Pujol, E. Quintana, E. Reverter, I. Rovira, P. Ruiz, E. Sandoval, S. Schneider, F. Seguí, O. Sibila, C. A. Soriano, D. Soy, M. Suárez, A. Téllez, ND. Toapanta, A. Torres, X. Urra.

Author contributions GP, AMA and EP contributed to the study conception and design. Material preparation, data collection and analysis were performed by GP, AMA, EH and MB. The first draft of the manuscript was written by GP and AM. MB, EH, LFQ, GMR, JM, PC, ES, RA, JF, JRB, AS, EP: interpreted the data, revised the manuscript, provided intellectual content and approved the final version.

Funding The authors received no financial support for the research, authorship, and/or publication of this article.

Code availability Not applicable.

\section{Compliance with ethical standards}

Conflict of interest The authors declare no conflict of interest.

Availability of data and material statement The datasets used and/or analyzed during the current study are available from the corresponding author on reasonable request.

Ethics approval The Institutional Ethics Committee approved the study with the identification code HCB/2020/0569. Informed consent was obtained from the patients or their legal representative, in accordance with the regulations of the ethics committee for patients with COVID19.

\section{References}

1. Callaway E (2020) Time to use the p-word? Coronavirus enters dangerous new phase. Nature. https://doi.org/10.1038/d4158 6-020-00551-1

2. Van Staden C (2020) COVID-19 and the crisis of national development. Nat Hum Behav 4(5):443-444

3. Ministry of Health; Government of Spain (2020). https://covid 19.isciii.es/. Accessed 20 May 2020

4. Zhang H, Penninger JM, Li Y, Zhong N, Slutsky AS (2020) Angiotensin-converting enzyme 2 (ACE2) as a SARS-CoV-2 receptor: molecular mechanisms and potential therapeutic target. Intensive Care Med 46(4):586-590

5. Letko M, Marzi A, Munster V (2020) Functional assessment of cell entry andreceptor usage for SARS-CoV-2 and other lineage B betacoronaviruses. Nat Microbiol 5:562-569
6. Liu Z, Xiao X, Wei X et al (2020) Composition and divergence of coronavirus spike proteins and host ACE2 receptors predict potential intermediate hosts of SARSCoV-2. J Med Virol. https ://doi.org/10.1002/jmv.25726

7. Hoffmann M, Kleine-Weber H, Schroeder S et al (2020) SARSCoV-2 cell entry depends on ACE2 and TMPRSS2 and is blocked by a clinically proven protease inhibitor. Cell 181(2):271-280

8. Guan W-J, Ni Z-Y, Hu Y et al (2020a) Clinical characteristics of oronavirus disease 2019 in China. N Engl J Med 382:1708-1720

9. Wang D, Hu B, Hu C et al (2020) Clinical characteristics of 138 hospitalised patients with 2019 novel coronavirus-infected pneumonia in Wuhan, China. JAMA 323(11):1061-1069

10. Huang C, Wang Y, Li X et al (2020) Clinical features of patients infected with 2019 novel oronavirus in Wuhan, China. Lancet 395:497-506

11. Chen N, Zhou M, Dong X et al (2020) Epidemiological and clinical characteristics of 99 cases of 2019 novel coronavirus pneumonia in Wuhan, China: a descriptive study. Lancet 395:507-513

12. Diao B, Wang C, Wang R et al (2020) Human kidney is a target for novel severe acute respiratory syndrome oronavirus 2 (SARS-CoV-2) infection. medRxiv. https://doi. org/10.1101/2020.03.04.20031120

13. Zhou F, Yu T, Du R et al (2020) Clinical course and risk factors for mortality of adult in patients with COVID-19 in Wuhan, China: a retrospective cohort study. Lancet 395:1054-1062

14. Cheng Y, Luo R, Wang $\mathrm{K}$ et al (2020) Kidney disease is associated with inhospital death of patients with COVID-19. Kidney Int 97:829-838

15. Chen $\mathrm{T}, \mathrm{Wu} \mathrm{D}, \mathrm{Chen} \mathrm{H}$ et al (2020) Clinical characteristics of 113 deceased patients with coronavirus disease 2019: retrospective study. BMJ 368:m1091

16. Hirsch JS, Ng JH, Ross DW et al (2020) Acute kidney injury in patients hospitalised with COVID-19. Kidney Int. https://doi. org/10.1016/j.kint.2020.05.006

17. Yang X, Yu Y, Xu J et al (2020) Clinical course and outcomes of critically ill patients with SARS-CoV-2 pneumonia in Wuhan, China: a single centered, retrospective, observational study. Lancet Respir Med 8:475-481

18. Arentz M, Yim E, Klaff L et al (2020) Characteristics and outcomes of 21 critically ill patients with COVID-19 in Washington state. JAMA 323(16):1612-1614

19. Ronco C, Reis T, Husaisn-Syed F (2020) Management of acute kidney injury in patients with COVID-19. Lancet Respir Med. https://doi.org/10.1016/S2213-2600(20)30229-0

20. Su H, Yang M, Wan C et al (2020) Renal histopathological analysis of 26 postmortem findings of patients with COVID-19 in China. Kidney Int. https://doi.org/10.1016/j.kint.2020.04.003

21. Lopes JA, Jorge S (2013) The RIFLE and AKIN classifications for acute kidney injury: a critical and comprehensive review. Clin Kidney J 6(1):8-14

22. Leveys A, Eckardt K-U, Tsukamoto Y et al (2005) Definition and classification of chronic kidney disease: a position statement from Kidney Disease: Improving Global Outcomes (KDIGO). Kidney Int 67:2089-2100

23. Guan W-J, Ni Z-Y, Hu Y et al (2020b) Clinical characteristics of coronavirus disease 2019 in China. N Engl J Med 382:1708-1720

24. Puelles VG, Lütgehetmann M, Lindenmeyer MJ et al (2020) Multiorgan and renal tropism of SARS-CoV-2. N Engl J Med. https:// doi.org/10.1056/NEJMc2011400

25. Varga Z, Flammer AJ, Steiger P et al (2020) Endothelial cell infection and endotheliitis in COVID-19. Lancet 395:1417-1418

26. Gaillard F, Ismael S, Sannier A (2020) Tubuloreticular inclusions in COVID-19-related collapsing glomerulopathy. Kindey Int. https://doi.org/10.1016/j.kint.2020.04.022 
27. Klok FA, Kruip MJHA, van der Meer NJM (2020) Incidence of thrombotic complications in critically ill ICU patients with COVID-19. Thromb Res 191:145-147

28. Tang N, Bai H, Chen X, Gong J, Li D, Sun Z (2020) Anticoagulant treatment is associated with decreased mortality in severe coronavirus disease 2019 patients with coagulopathy. J Thromb Haemost 18(5):1094-1099

29. Mohamed MMB, Lukitsch I, Torres-Ortiz AE et al (2020) Acute kidney injury associated with coronavirus disease 2019 in urban New Orleans. Kidney360. https://doi.org/10.34067/KID.00026 52020
30. Richardson S, Hirsch JS, Narasimhan M, Crawford JM, McGinn T, Davidson KW, Barnaby DP, Becker LB, Chelico JD, Cohen SL, Cookingham J (2020) Presenting characteristics, comorbidities, and outcomes among 5700 patients hospitalized with COVID-19 in the New York City Area. JAMA 323(20):2052-2059. https:// doi.org/10.1001/jama.2020.6775

Publisher's Note Springer Nature remains neutral with regard to jurisdictional claims in published maps and institutional affiliations.

\section{Affiliations}

\section{Gaston J. Piñeiro' ${ }^{1}$ - Alicia Molina-Andújar ${ }^{1} \cdot$ Evelyn Hermida $^{1} \cdot$ Miquel Blasco $^{1} \cdot$ Luis F. Quintana $^{1}$. Guido Muñoz Rojas ${ }^{2}$. Jordi Mercadal ${ }^{2} \cdot$ Pedro Castro $^{3} \cdot$ Elena Sandoval $^{4} \cdot$ Rut Andrea $^{5} \cdot$ Javier Fernández $^{6}$. Joan Ramon Badia ${ }^{7}$ Alex Soriano ${ }^{8}$. Esteban Poch ${ }^{1}$ on behalf of the Hospital Clínic Critical Care COVID-19 working group (CCCC)}

1 Nephrology and Kidney Transplantation Department, Hospital Clínic, IDIBAPS, University of Barcelona and REDinREN, Villarroel 170, 08036 Barcelona, Spain

2 Surgical Intensive Care Unit, Anesthesiology Department, Hospital Clínic, IDIBAPS, Univesitat de Barcelona, Barcelona, Spain

3 Intensive Care Unit, Internal Medicine Department, Hospital Clínic, IDIBAPS, Univesitat de Barcelona, Barcelona, Spain

4 Cardiovascular Intensive Care Unit, Cardiovascular Surgery Department, Hospital Clínic, IDIBAPS, Univesitat de Barcelona, Barcelona, Spain
5 Acute Cardiac Care Unit, Cardiology Department, Hospital Clínic, IDIBAPS, Univesitat de Barcelona, Barcelona, Spain

6 Intensive Care Unit, Hepatology Department, Hospital Clínic, IDIBAPS, Univesitat de Barcelona, Barcelona, Spain

7 Respiratory Institute, Hospital Clínic, IDIBAPS, Univesitat de Barcelona, Barcelona, Spain

8 Infectious Diseases Department, Hospital Clínic, IDIBAPS, Univesitat de Barcelona, Barcelona, Spain 